\title{
Your Memory Is Working Against You: \\ How Eye Tracking and Memory Explain Habituation to Security Warnings
}

\begin{abstract}
Security warnings are critical to the security of end users and their organizations, often representing the final defense against an attack. Because warnings require users to make a contextual judgment, it is critical that they pay close attention to warnings. However, research shows that users routinely disregard them. A major factor contributing to the ineffectiveness of warnings is habituation, the decreased response to a repeated warning. Although previous research has identified the problem of habituation, the phenomenon has only been observed indirectly through behavioral measures. Therefore, it is unclear how habituation develops in the brain in response to security warnings, and how this in turn influences users' perceptions of these warnings.

This paper contributes by using eye tracking to measure the eye movement-based memory (EMM) effect, a neurophysiological manifestation of habituation in which people unconsciously scrutinize previously seen stimuli less than novel stimuli. We show that habituation sets in after only a few exposures to a warning and progresses rapidly with further repetitions. Using guidelines from the warning science literature, we design a polymorphic warning artifact which repeatedly changes its appearance. We demonstrate that our polymorphic warning artifact is substantially more resistant to habituation than conventional security warnings, offering an effective solution for practice. Finally, our results highlight the value of applying neuroscience to the domain of information security behavior.
\end{abstract}

\section{Keywords}

NeuroIS; eye tracking; security warnings; habituation; behavioral information security 


\section{Introduction}

Security warnings are critical to the security of end users and their organizations. Warnings often represent the last line of defense, the final barrier, between an attack and its intended target. Security systems would ideally detect and prevent a threat without user intervention. However, many

5 situations require the user to make a security judgment based on contextual factors. For example, consider the 2011 breach of RSA Security LLC, which led to the compromise of Lockheed Martin and other aerospace and defense companies $[1,2]$. It is telling that the RSA breach could be traced back to a malicious email attachment that was sent to an RSA employee. Because the email client software could not definitively determine the threat of the attachment, a security warning was triggered which required human judgment [3; see Figure 1].

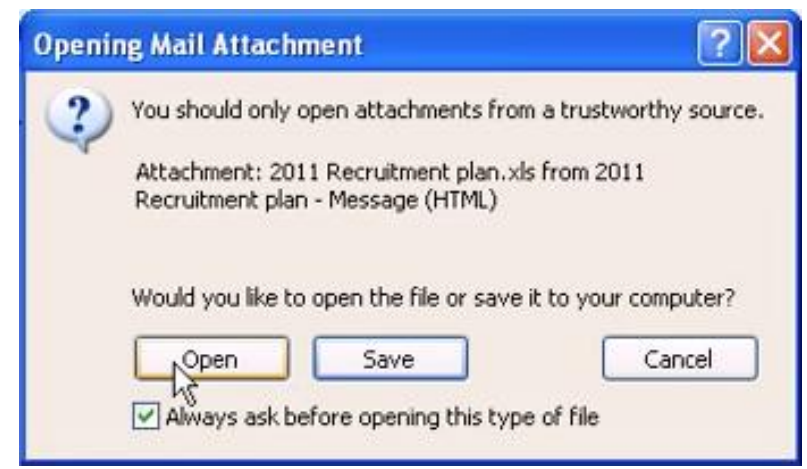

Fig. 1. The actual warning message that immediately preceded the installation of malware that led to the breach of RSA Security [3].

Although the employee's failure to heed this warning may not have been entirely to blame for these security incidents, it is clear that users' responses to warnings play a critical role in organizations' security. As a result, researchers have actively sought to understand how users perceive and interact with security warnings [4].

Unfortunately, research has shown that security warnings are disappointingly ineffective [5-7]. One frequently cited factor that influences the effectiveness of warnings is habituation, which is the 
"decreased response to repeated stimulation" [8, p. 419]. Although frequently cited as a problem in research on warnings, habituation is often inferred, rather than examined directly [9, 10]. For example, a study examining security warnings found that $42 \%$ of participants were not aware of having interacted with security permission dialogs before installing an Android app on their devices

5 [11]. In another study, Sotirakopoulos et al. [6] observed that some participants clicked through security warnings during a task and later reported that they had not seen any security warnings. Habituation would seem a likely explanation for these behaviors. However, without a direct measure of habituation, it is unclear (1) how it influences our perceptions of warnings, and (2) whether new warning designs are effective in reducing its occurrence.

In this paper, we contribute by using the methods and theory of neuroscience applied to information systems (NeuroIS) to directly measure the process of habituation. In doing so, we respond to the call of Crossler et al. [12] and Luo and Zhdanov [13] for the application of NeuroIS methods to yield fresh insights into information-security behaviors. Specifically, memory researchers have discovered a pervasive phenomenon in which people unconsciously pay less attention to images

15 that they have previously viewed. This manifestation of habituation, termed the eye movement-based memory (EMM) effect [14], is apparent in fewer eye-gaze fixations and less visual sampling of regions of a visual stimulus. Further, people's attention decreases systematically with repeated viewings, even when people do not consciously recognize that they have seen an image before [15]. For these reasons, the EMM effect is a robust means of directly observing the phenomenon of 20 habituation to security warnings and evaluating warning designs intended to reduce its occurrence. Accordingly, our research questions are

RQ1. How does habituation affect perceptions of security warnings?

RQ2. How can security warnings be designed to be more resistant to habituation? 
We conducted a laboratory experiment using the method of eye tracking to measure the EMM effect as users viewed a series of security warnings. In addition, with guidance from the warning science literature [16], we developed a user-interface (UI) design artifact [17], a polymorphic warning that successively changes its appearance with each exposure as a way to sustain users'

5 attention and reduce habituation. We compared the rate of habituation to polymorphic warnings with that of conventional, static warnings whose appearance does not change. The results of the analysis of eye-gaze fixation data demonstrated that our polymorphic warning is substantially more resistant to habituation than static warnings.

Our findings provide several contributions. First, we show that the EMM effect can be used as

10 a direct neurophysiological measure of habituation to security warnings, and we depict how habituation progresses over time. Second, we develop a UI-design artifact - a polymorphic warning - comprising nine graphical variations derived from the warning science literature. Third, using eye tracking, we empirically demonstrate the effectiveness of our polymorphic warning design in reducing habituation vis-à-vis conventional warnings. Finally, this research illustrates the value of

15 applying NeuroIS to the domain of behavioral information security.

The paper proceeds as follows. We next review the relevant literature on warnings, habituation, and the EMM effect. After the development of our hypotheses, we outline our eye-tracking methodology and our experimental design, along with our analysis. We conclude with a discussion of our contributions and their implications for research and practice.

\section{2. Literature Review}

\subsection{What is Habituation?}

Habituation is "decreased response to repeated stimulation" [18, p. 419]. Habituation [8, p. 419] is believed to be ubiquitous in the animal kingdom, having been found "in every organism studied, from single-celled protozoa, to insects, fish, rats, and people" [19, p. 125]. In contrast to 
associative learning, in which a response to stimulus is associated with another stimulus (e.g., Pavlovian conditioning), habituation is a form of nonassociative learning, because an organism undergoing habituation adjusts the way in which it responds to a stimulus without pairing it with another stimulus, such as a specific consequence [20].

Habituation is an important survival mechanism because it allows organisms to filter out stimuli in the environment that are not relevant, thus conserving energy to respond to stimuli that predict things that are good or bad for survival [21]. As a filtering mechanism, habituation is different from sensory or motor fatigue [19]. That is, habituation occurs not because an organism tires of a stimulus, but because the organism filters it out as unimportant. Not surprisingly, humans

10 also exhibit habituation to a wide variety of stimuli-visual, auditory, and others — and it is evident as early as infancy [22]. In the context of this study, we conceptualize habituation in terms of diminished attention to repeated security warnings [19].

\subsection{Habituation and Habit Compared}

Although the words have the same Latin root, the constructs of habituation and habit are very different. Habit is defined as "learned sequences of acts that have become automatic responses to specific cues, and are functional in obtaining certain goals or end-states" [23, p. 104]. Further, habits are "created by frequently and satisfactorily pairing the execution of an act in response to a specific cue" $[24$, p. 1314]. These descriptions show that habit occurs at the behavioral level and is a form of associative learning in which behaviors are associated with specific outcomes.

Habituation, in contrast, occurs at the neurobiological level [25]; it is a form of nonassociative learning in which an organism filters out stimuli that in the past have not led to relevant outcomes [26]. Further, habituation does not require subsequent behavior but occurs involuntarily, that is, without conscious awareness. Although habitual behavior is also relevant to security warnings, we restrict our focus to habituation in this study. 


\subsection{Habituation and Security Warnings}

A number of experiments have pointed to the role of habituation in the failure of information security warnings [see 27, 28]. For example, a laboratory study conducted by Sunshine et al. [5] observed that participants remembered their responses to previous security warnings and applied them to other websites even if the level of risk involved had changed. These results are mirrored by

those in the field, including a study [9] in which users were rapidly exposed to a confirmation dialog message. After a period of 2.5 minutes and a median of 54 exposures to the dialog message, only $14 \%$ of the participants recognized a change in the content of the confirmation dialog in their control (status quo) condition. Akhawe and Felt found that in approximately $50 \%$ of SSL warnings, users decided to click through in 1.7 seconds or less, a finding that "is consistent with the theory of warning fatigue" [7, p. 270]. Although this literature is valuable for highlighting the problem of habituation, the studies examined habituation indirectly by observing its influence on security behavior $[9,10]$. As a result, these studies did not provide a means to directly measure the mental process of habituation, and the lack of such a means limits our ability to design security messages

15 and interventions that directly address the phenomenon. Wogalter states that "habituation can occur even with well-designed warnings, but better designed warnings with salient features can slow the habituation process. Where feasible, changing the warning's appearance may be useful in reinvigorating attention switch previously lost because of habituation" [29, p. 55]. Other studies such as Bravo-Lillo et al. [30] support this argument by testing the effects of warning attractors, which are manipulations of the user interface to draw attention to salient information in the warning dialogs, suggesting that habituation can be reduced through UI design.

In line with this reasoning, Brustoloni and Villamarín-Salomón [10] proposed polymorphic information security warnings, which change the form of user inputs to prevent habituation. While acknowledging that the design space for polymorphic warnings is immense, they considered only one 
example of polymorphism: randomizing the order in which options are displayed on a warning. They found in a laboratory experiment that participants who received the polymorphic warning opened risky email attachments significantly less often than those who received the conventional warning.

\subsection{Eye Tracking}

We measure eye movements as a means of accessing the "black box" of cognition. Eyetracking tools can precisely measure eye position and eye movement [31], including eye fixation, pupil dilation, gaze duration, and areas of interest [32]. The method allows researchers to understand what participants pay attention to, and therefore what is available to perception $[33,34]$. Eye-tracking tools provide data such as heat maps to indicate the percentage of time spent gazing at any particular area (see Fig. 2).
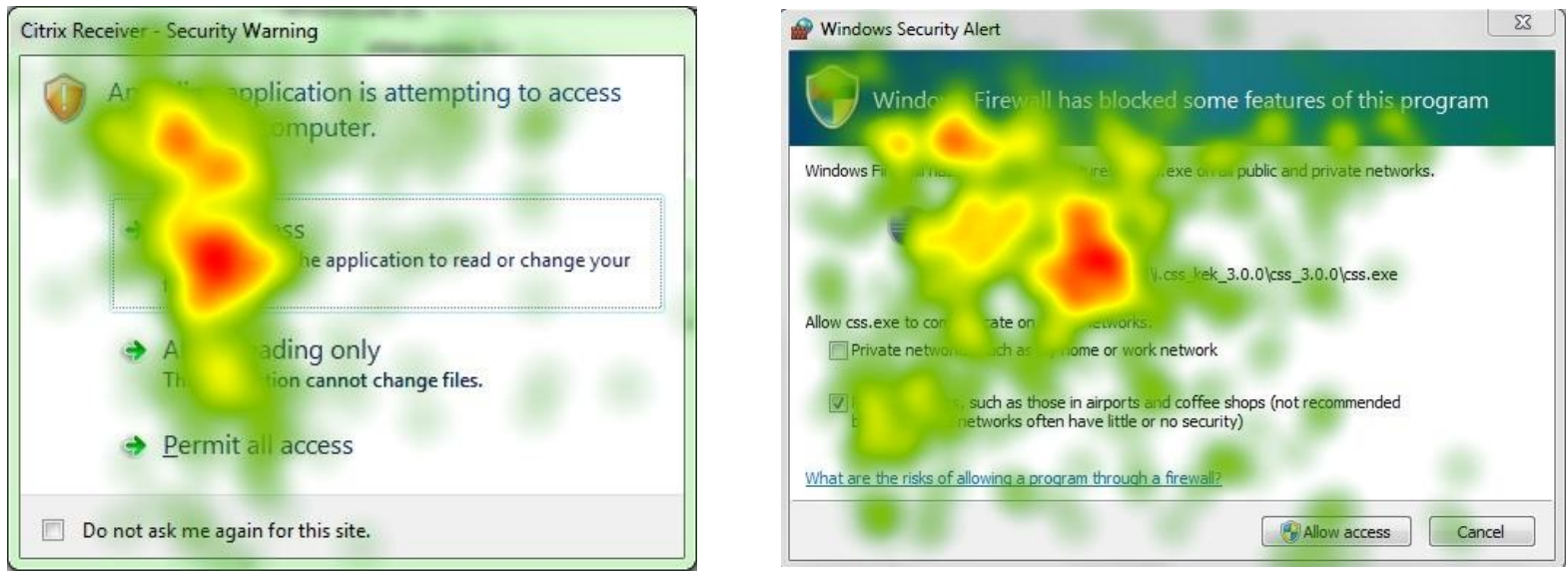

Fig. 2. Heat maps for images of two security warnings.

Eye tracking and other NeuroIS tools have recently received attention due to their ability to complement existing sources of data with data captured directly from the human body. Distinct from other NeuroIS tools, eye tracking's most notable advantage is its ability to measure human visual activities with a high level of accuracy and temporal precision. This information is not possible

15 through self-reported means because people are not able to perfectly recall or are not fully conscious of what they saw, where they looked, and in what order they looked [35]. 
Eye-movement behavior provides an important insight into cognitive processes that may not be available to conscious introspection. For example, when reading a passage of text, most people's eyes do not move smoothly from left to right across the page. Rather, viewers make a series of brief fixations and jumps ("saccades") of about 7-9 characters. This means that words shorter than 7-9

5 characters are routinely skipped and may not be remembered when later tested. The information that is not in the center of the fixation is not directly perceived, as can be demonstrated by altering all the text on a page except the text at the center of the fixation. When these changes are made during the saccade, participants do not notice, even when they are aware that the changes are occurring [32]. When reading a difficult passage, we make reverse saccades to reread previous material, often without noticing. For these reasons, eye-tracking devices have been a staple among neurophysiological tools for many years [see 14, 34, 36].

Eye tracking has also been used in IS studies. For example, Goldberg et al. [37] used an eyetracking device in a human-computer interaction scenario to evaluate software design and user interaction with the program. These researchers also evaluated the use of eye tracking in web search

15 tasks to create more-effective web portals. In another study, Cyr et al. [38] used eye tracking to measure the effects of human images on website appeal and trust. Meservy et al. [39] demonstrated using eye movement data that knowledge seekers filter information in online forums using peripheral cues such as source expertise and validation. Léger et al. [40] synchronized eye tracking and electroencephalography data to precisely measure users' neural activity at the time they first perceive 20 and process a visual stimulus. Researchers in human-computer interaction [41] and computer usability studies [42] have successfully used eye tracking to compare the usability or effectiveness of a variety of screen interfaces. Likewise, this study uses an eye-tracking device to measure and interpret the eye movements of participants and the overt classification decisions they make. 


\subsection{Eye Movement as an Index of Memory}

Eye movements have been used extensively as an index of cognitive processing in both cognitive psychology [43] and cognitive neuroscience [44]. In addition, neuroscientists have established the utility of tracking eye movements as a direct measure of habituation and an indirect measure of memory [36]. Research has shown that the EMM effect is an obligatory consequence of previous exposure that is observed even when people do not consciously recognize that they have seen an image before [34]. In their review of eye movement and memory, Hannula et al. observed that the results of EMM studies show that "the very nature of perceptual processing is altered as a result of our prior experiences" $[15$, p. 14]. When presented with a repeated stimulus, participants tend to make fewer fixations and spend less time looking at the repeated stimulus. In a series of experiments, Cohen et al. [for a review, see 15] have demonstrated that when elements of a scene are rearranged, participants fixate more on the changed region, even in the absence of conscious awareness of any change. For example, in Ryan et al. [14], participants were presented with novel, repeated, or manipulated scenes and their eye movements were tracked while they investigated the scenes. They demonstrated that the participants fixated more and spent more time looking at regions of the images where manipulations had occurred between the initial presentation and the presentation of the manipulated images. This effect was independent of conscious awareness.

In contrast, Smith and Squire [36] found that eye movements into and out of critical regions are dependent on an individual's awareness that a region has been altered. Study participants who were unaware of the alteration viewed the image similarly to how they viewed it before they were shown the manipulated version. Thus, they did not fixate on those areas unless they were conscious of a change occurring. Research on the relationship between conscious awareness and experiencedependent changes in eye movements (i.e., the EMM effect) is ongoing [15]. These experiments inform the present study by (1) providing evidence for an effect of memory on eye movements 
(perhaps with or without conscious awareness) and by (2) demonstrating how to measure the EMM effect.

\section{Theory and Hypotheses}

According to the EMM literature, people's previous viewing experience influences the proportion of fixations and viewing time spent on manipulated regions, and the number of transitions into and out of manipulated regions. This effect is evident in both the number of eye fixations and the number of regions sampled in an image [34]. Interestingly, with repeated viewings, attention decreases systematically [45]. This indicates that the EMM effect is not a one-time event, but instead an ongoing process in which the brain reduces the amount of time and effort required to visually extract information from an image by leveraging mental models (i.e., memories) of previous viewings [46]. With repeated exposure, the mental models become increasingly available, requiring less visual sampling of an image [45].

The EMM effect is further explained by the dual-process theory of habituation of the field of neurobiology. It explains how the EMM effect can be a valid measure of habituation [26]. This

15 theory proposes that when people see a stimulus, they generate a mental model (e.g., memory) of the stimulus. When people see a stimulus in the future that matches this mental model, a habituation process occurs in which people give less attention to the warning and instead rely more on the mental model to interpret and respond to the stimulus. This results in more efficient mental processing, but also decreased eye-gaze fixations and visual sampling of the stimulus (i.e., the EMM effect) [14]. If

20 the stimulus does not match the mental model, a sensitization process occurs that counters habituation. When sensitization occurs, people rely less on previous mental models and instead visually inspect the warning to generate a new mental model.

Drawing upon the EMM literature, we derive four hypotheses that explain how habituation affects visual processing of security warnings. We first consider the warning as a whole, including all 
of its visual elements (border, symbols, text, etc.). Then we consider only the text portion of a warning. For the entire warning visual area, many warning styles follow similar design principles; for instance, indicators of alarm including bright red colors, exclamation marks, bolded text, and two buttons for choosing whether to heed or ignore the warning. As users are exposed to repeated warnings, the dual-process theory of habituation explains that users will form mental models of these warnings in memory. When people see the warning again in the future, they rely more on these mental models rather than visually examining the stimulus $[8,47]$. As a result, people will visually inspect and process the warning less, because they rely more on the mental model using multiple memory systems [e.g., 48, 49, 50]. This should in turn be associated with decreased visual processing according to the EMM effect. Therefore, we hypothesize:

Hypothesis 1. Warning gaze duration will decrease over successive viewings per subject.

However, a polymorphic warning whose visual appearance constantly changes should prevent users' from relying on the brain's mental models. When a warning changes its appearance, it conflicts with the mental model of previous warnings. The dual-process theory of habituation 15 explains that this results in a sensitization process that counters habituation $[8,47]$. As a result, people do not rely on this mental model, but rather give attention and visually inspect the polymorphic warning. Computational and theoretical models of memory in the brain propose that even small changes in a stimulus may result in a novelty signal resulting in the subject treating the stimulus as new and thereby not relying on previous mental models [e.g., 51, 52]. A growing body of 20 empirical evidence supports these models' predictions [c.f. 53]. This should in turn be associated with a smaller decrease in visual processing according to the EMM effect for polymorphic warnings compared to static warnings. Accordingly, we predict:

Hypothesis 2. Warning gaze duration will decrease more rapidly when viewing static warnings as compared to polymorphic warnings. 
We next consider the textual element of a warning, which is particularly important in order for a user to act on a warning message. Failure to comprehend a warning's text, and to be motivated by the text, will prevent a user from performing the desired security behavior. If increased visual attention occurs for the overall warning but not for the textual component in isolation, then

5 employing polymorphic warnings could be considered an incomplete remedy for the problem of inattention to warnings.

We hypothesize that polymorphic warnings will garner greater attention to a warning's text, although only the visual presentation of the warning has changed and not the text itself. This is consistent with the dual-process theory of habituation $[8,47]$. When a warning does not change

10 appearance (e.g., a static warning) people rely on the mental model to interpret the warning, including recalling the warning purpose or text. However, when a warning changes its appearance (e.g., a polymorphic warning), it causes sensitization that conflicts with the existing mental model. As a result, people will visually examine the warning to create a new mental model. As a part of this examination, people will also read the text to discover the meaning of the warning and store that in

15 memory rather than relying on previous mental models of past warnings. Thus, the polymorphic warning should prompt an increase in the visual processing of the text as compared to static warnings.

Our hypotheses for the textual element are parallel to the hypotheses for the overall warning (Hypothesis 1 and Hypothesis 2). First, the textual message component of warnings will likely carry

20 common elements across different warning manifestations, so repeated exposure to warnings will lead to a robust mental model, and therefore a lower level of visual processing according to the EMM-effect principles. Second, the rate of decrease in visual processing will be slower for polymorphic warning manifestations than for static ones because it conflicts with existing mental models of warnings, and therefore garners visual exploration of the warning. Accordingly, we hypothesize: 
Hypothesis 3. Warning text gaze duration will decrease over successive viewings per subject.

Hypothesis 4. Warning text gaze duration will decrease less rapidly when viewing polymorphic warnings as compared to static warnings.

\section{Methodology}

To test our hypotheses, we conducted a laboratory experiment using eye tracking. Eye-tracking technology is key to our study for four reasons. First, NeuroIS methods [35] such as eye tracking excel at capturing "hidden (automatic or unconscious) mental processes (e.g., habits, ethics, deep emotions) that are difficult or even impossible to measure with existing measurement methods and tools" [54, p. 688]. Habituation is one such process because it is automatic and fundamentally occurs

10 at the neurobiological level [50]. Therefore, people are likely not fully aware of the extent of their habituation to warnings. Second, security warnings are visual stimuli that require attention to the details of their appearance and message. Eye tracking can fully capture users' visual inspection of warnings. Third, habituation and decisions to respond to warnings occur very quickly [9]. With temporal precision in the tens of milliseconds, eye tracking is well suited to examine habituation to visual stimuli. Finally, eye tracking is unobtrusive compared to other NeuroIS methods because it allows the capture of eye movement data as participants naturally use a computer.

Our experiment utilized a within-subject design in which participants were randomly assigned to view variations of either polymorphic or static warnings. We then explored the number of fixations over the (1) entire warning and (2) the warning text across multiple viewings of warnings to gauge habituation via the EMM effect. We also captured eye movements during participants' visual processing of the warnings. By analyzing the eye movement data, we were able to gauge whether the EMM effect occurs in the context of processing security warnings. This in turn indicated the extent to which individuals habituate to warnings over repeated exposures. 


\subsection{The Polymorphic Warning-a UI-Design Artifact}

To reduce the occurrence of habituation, we developed a polymorphic warning - a UI-design artifact [17]. To do so, we drew upon the warning science literature to develop nine graphical variations of a warning dialog expected to capture attention [16]. Our polymorphic warning artifact rotated through the graphical variations with each subsequent exposure. Each graphical variation was chosen based on support from the warning science literature that it should attract increased attention. Table 1 lists each variation with its related references, and Fig. 3 depicts each variation for one of the 20 warnings presented in our experiment. While each of these variations has been shown to draw attention, users could still become habituated to any one of these variations. Therefore, our polymorphic warning design rotates through the variations to trigger the sensitization process explained by the dual-process theory of habituation, thereby reducing habituation.

\section{Table 1}

Polymorphic variations and their support from the warning science literature.

\section{Text Appearance Support}

Color of text (red text)

Braun et al. [55]; Laughery et al. [56]

Highlighting of text (yellow highlighting) Strawbridge [57]; Young and Wogalter [58]

\section{Message Content}

Pictorial symbols (an exclamation point)

Signal word ("Attention")

Warning Appearance

Color (red background)

Contrast (white on black)

Ordering of options (reordered)

Size (large)

\section{Support}

Kalsher et al. [59]; Sojourner and Wogalter [60]

Kalsher et al. [61]; Silver and Wogalter [62]

Support

Kalsher et al. [61]; Silver and Wogalter [62]

Braun and Silver [63]; Rudin-Brown et al. [64]

Sanders and McCormick [65]; Young [66]

Vigilante and Wogalter [67]; Wogalter and Vigilante [68]
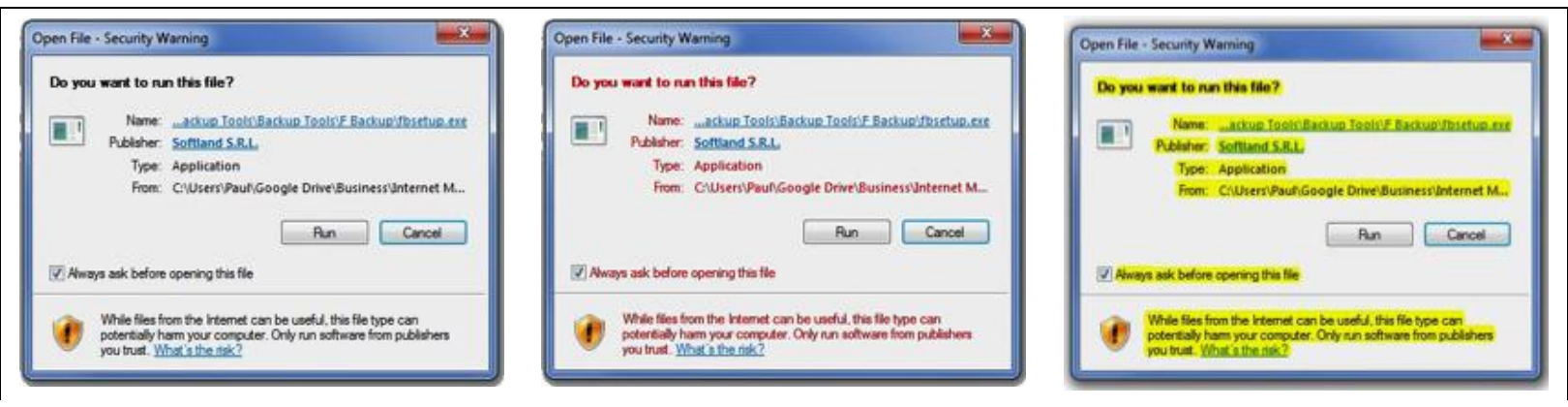
(a) Original warning screenshot



(d) Signal word variation



(g) Color variation (b) Color of text variation

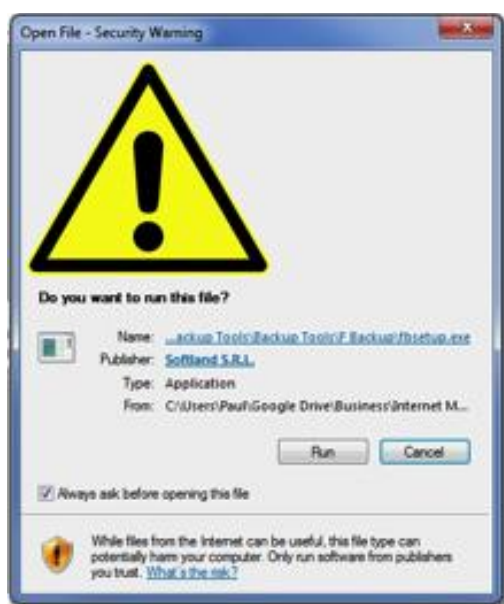

(e) Pictorial signals variation

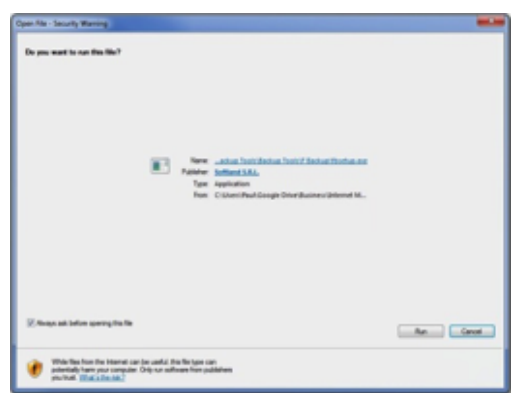

(h) Size variation (3X larger) (c) Highlight of text variation

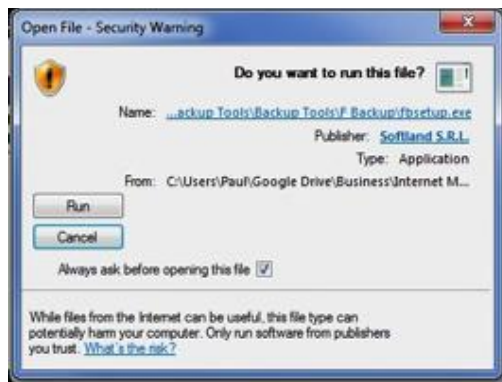

(f) Ordering of options variation

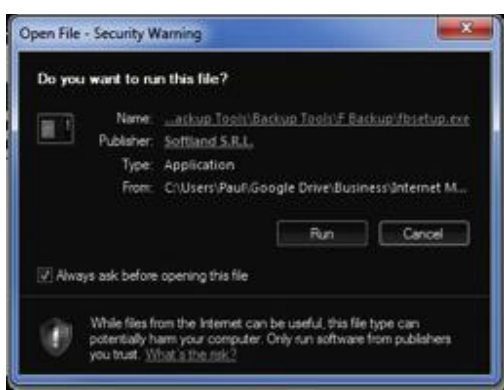

(i) Contrast variation

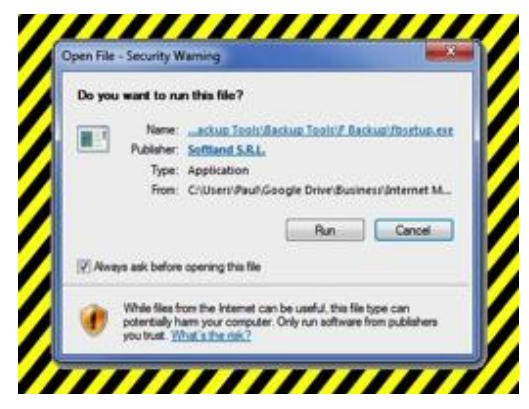

(j) Border variation

Fig. 3. Polymorphic warning design variants.

\subsection{Experimental design}

The university Institutional Review Board (IRB) approved our experimental protocol.

Participants were given an informed consent form to sign prior to the experiment. After the experiment, participants were debriefed. We used a Tobii T120 (see Fig. 4) to measure the EMM

5 effect. The eye tracker can track participants' eye movement with or without corrected vision 
(contact lenses and glasses), so we did not need to exclude any participants based on eyesight.

Participants were instructed to sit in front of the Tobii monitor. Tobii software was used to calibrate the machine to each participant. If there was an error, the participant was resituated and recalibrated. If the participant was unable to calibrate (due to severe ametropia or other abnormal refractive conditions) we would have excluded that participant [see 69, p. 109]. However, we did not encounter any subjects who needed to be excluded. The calibration process took approximately five minutes.

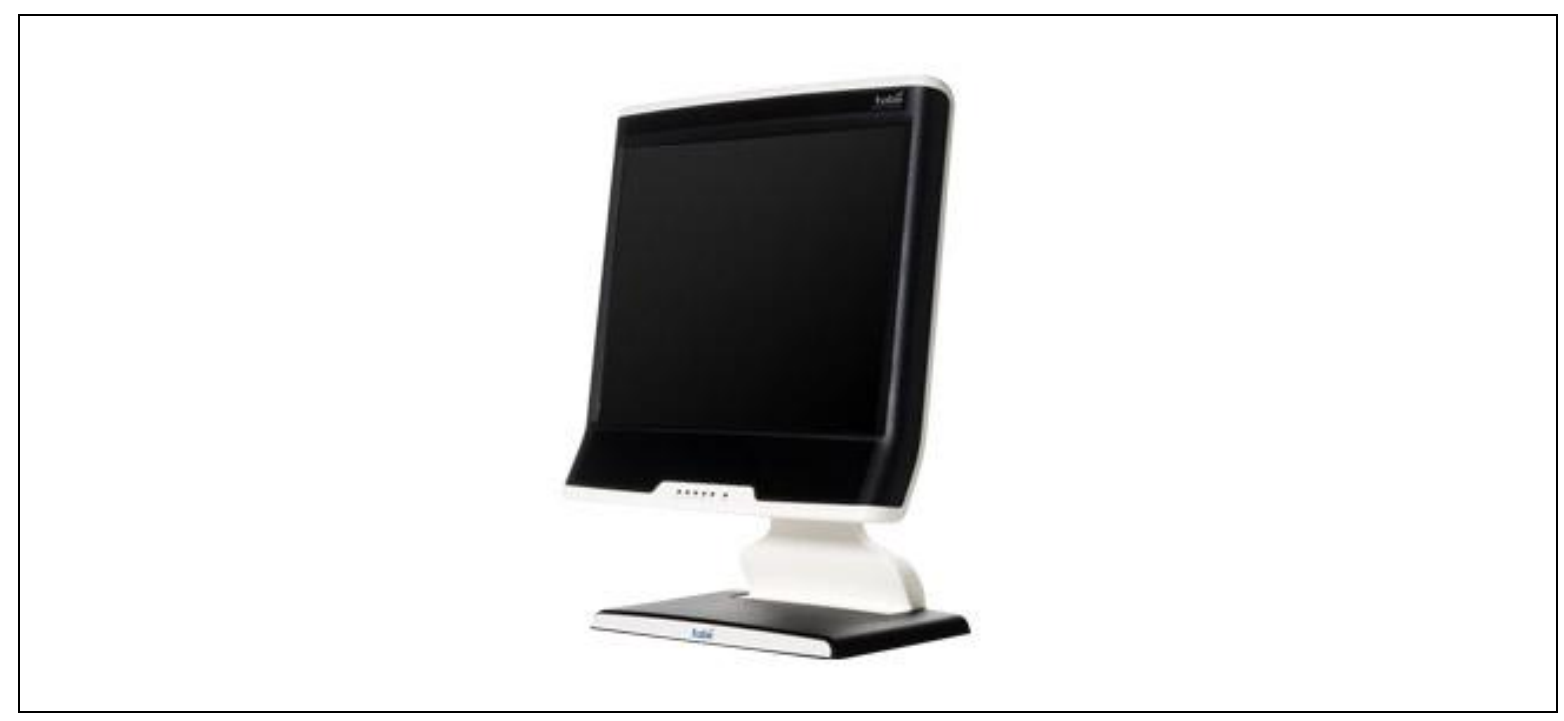

Fig. 4. Tobii T120 eye tracker with integrated monitor.

Next, participants were presented with 20 warnings. For each subject, these warnings were randomly and evenly assigned between the polymorphic and static treatments. During the experiment, both polymorphic and static images were repeated 10 times. For the polymorphic treatment, after the original warning, repetitions of the warning adopted one of the nine graphical variations listed in Table 1. Thus, participants viewed a total of 200 warning exposures (20 warnings $\times 10$ repetitions). The order of presentation for the entire pool of warning exposures was randomized to control for ordering effects.

To ensure that participants were actively engaged in the study, they were instructed to examine each warning carefully and assess whether the warning was: (1) novel within the study context, (2) 
similar to or a modified version of a previous image, or (3) identical to other images within the study (see [27]). The warnings were self-paced, meaning participants could control how long they viewed each image before proceeding. This was done to mimic real life, in which people choose how long to view a warning before dismissing it. The entire experiment lasted 20-40 minutes, depending on how

5 quickly participants completed the categorization task.

After viewing all of the warnings, we administered a postexperiment survey to capture demographic and other control variables. To ensure manipulation validity [70], the posttest survey included a manipulation-check question that displayed a polymorphic warning rotating through its graphical variations. Participants were asked, "During the experiment, did you notice that some of

10 the warnings changed their appearance like the above image?" Fully 127 of our 132 participants reported that they had noticed the experimental treatment, giving us assurance that the subjects were successfully manipulated overall. However, following Straub et al., we elected to retain participants who reported that they were not manipulated to provide "a more robust testing of the hypotheses" [70, p. 408].

\subsection{Participants}

We pilot tested our experimental design with 20 participants. After making adjustments, we ran the final version of the experiment and collected usable data from 132 participants. Students were recruited from a large private university in the United States, and they received course credit for participating. Participants' ages ranged 18-46, with a mean of 21.48 years. Of the 132 participants,

$2058(44 \%)$ were female. Typical of eye tracking, occasionally participants' movement caused the eye tracker to temporarily lose its focus on their eye. These instances were not included in the analysis. As a result, we had 23,359 usable observations. 


\section{Analysis}

Our eye tracker was configured to record fixations at a rate of $60 \mathrm{hertz}$, capturing millions of eye movements from participants as they viewed the warnings. The number of fixations is roughly equivalent to the number of $16.66 \mathrm{~ms}$ time periods that the participant was gazing at the area of interest. Fig. 5 and Fig. 6 plot the Lowess curve (a plotting method for fitting a smooth curve between two variables) for the number of fixations over time on the warning and warning text, respectively. Prior to the analysis, a square-root transformation was performed on the number of fixations (a typical transformation for counts) to increase the linearity of the trend lines.

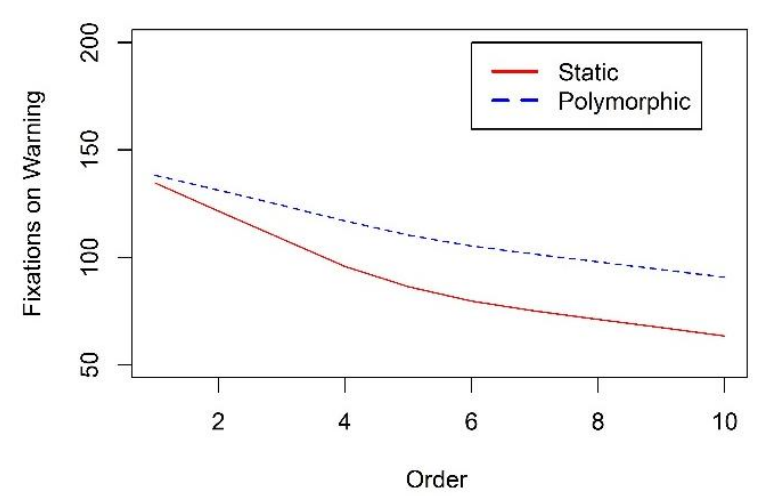

Fig. 5. Interaction between warning type and repetition number (order) for fixations on warning

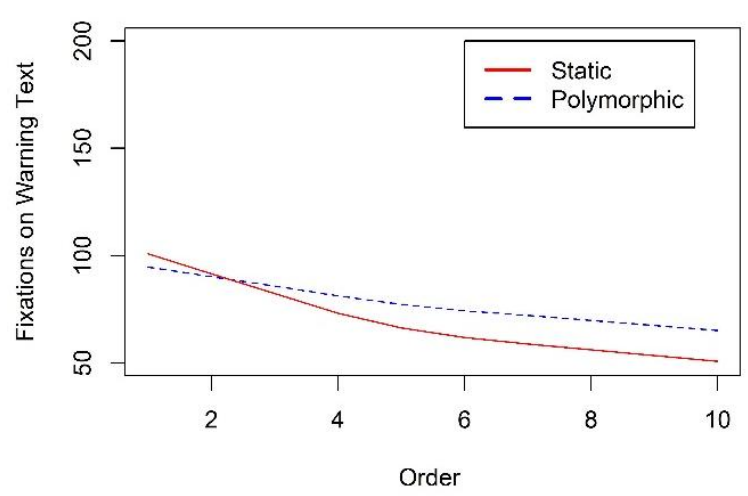

Fig. 6. Interaction between warning type and repetition number (order) for fixations on warning text

To test the hypotheses, we specified two linear mixed-effects models using the lme4 package in and one with warning text fixations as the dependent variable (to test Hypothesis 3 and Hypothesis 4). For each model, we included a random factor for the participant id, which controlled for natural fixation differences among participants (i.e., it allowed participants to have their own intercepts). We also included a random factor for warning id, which controlled for the possibility that some warnings may naturally garner more fixations than others. To test for habituation over successive viewings, we 
included a fixed factor for warning repetition, indicating whether it was the first, second, or $n$th repetition of a given warning. A negative significant effect of warning repetition indicates that fixations decrease across reputations. We included whether the warning was polymorphic $(1=$ polymorphic, $0=$ static) as a fixed factor. We also included an interaction term between polymorphic

5 and warning repetition, to test if habituation is slower for polymorphic warnings (i.e., if fixations decrease less rapidly for polymorphic warnings). Finally, we included a fixed factor for overall stimulus display count (i.e., the $n$th of 200 warnings overall). This controlled for effects of fatigue due to the number of warning participants saw.

The analysis for the model with warning fixations as the dependent variable is shown in Table 2. The analysis supports our hypotheses. The fixed factor of stimulus display count was significant, indicating that fatigue did have an effect. However, the estimate of warning repetition was significantly negative $\left(\beta=-0.232, t=-8.423_{(23,351)}, p<.001\right)$, indicating that gaze duration decreased over successive viewings per subject (Hypothesis 1 supported). This demonstrated that habituation to single repeated warning stimulus had a significant effect over and above that of overall fatigue. In

15 addition, the interaction between polymorphic warnings and warning repetitions was significantly positive $\left(\beta=0.106, t=5.881_{(23,351)}, p<.001\right)$, indicating that warning gaze duration decreases more slowly for polymorphic warnings (Hypothesis 2 supported). The $R^{2}$ of the model was .442 .

Table 2

Results for warning fixations model.

\begin{tabular}{lrrrr}
\hline & Estimate & Std. error & $t$ value & $p$ value \\
\hline Intercept & 12.959 & 0.298 & 43.521 & $<.001$ \\
Polymorphic & 0.261 & 0.112 & 2.325 & $<.020$ \\
Stimulus display count & -0.028 & 0.003 & -11.213 & $<.001$ \\
Warning repetition & -0.232 & 0.028 & -8.423 & $<.001$ \\
Polymorphic * Warning repetition & 0.106 & 0.018 & 5.881 & $<.001$
\end{tabular}

The analysis for the model with warning text fixations as the dependent variable is shown in Table 3. This analysis likewise supports our hypotheses. As before, the fixed factor of stimulus 20 display count was significant, indicating that fatigue did have an effect. However, the estimate of 
warning repetition was negative $\left(\beta=-0.244, t=-9.319_{(23,351)}, p<.001\right)$ indicating that gaze duration decreases over successive viewings per subject (Hypothesis 3 supported). Again, this demonstrated that habituation to a single repeated warning stimulus had a significant effect over and above that of overall fatigue. In addition, the interaction between polymorphic warnings and warning repetitions was significantly positive $\left(\beta=0.118, t=6.872_{(23,351)}, p<.001\right)$, indicating that warning gaze duration decreases more slowly for polymorphic warnings (Hypothesis 4 supported). The $R^{2}$ of the model was .416.

\section{Table 3}

Results for warning text fixations model.

\begin{tabular}{lrrrr}
\hline & Estimate & Std. error & $t$ value & $p$ value \\
\hline Intercept & 11.202 & 0.323 & 34.650 & $<.001$ \\
Polymorphic & -0.359 & 0.107 & -3.362 & $<.001$ \\
Stimulus display count & -0.018 & 0.002 & -7.746 & $<.001$ \\
Warning repetition & -0.244 & 0.026 & -9.319 & $<.001$ \\
Polymorphic * Warning repetition & 0.118 & 0.017 & 6.872 & $<.001$ \\
\hline
\end{tabular}

Because of the large $n$ resulting from the eye tracking data, we performed a supplemental analysis to assess the effect size of polymorphic warnings alone. We performed $t$-tests comparing the amount of change (in the number of fixations) between static and polymorphic warnings from the first time a warning was shown to the tenth time. First, for fixations over the entire warning, static warnings decreased 141.62 fixations on average from the first warning to the tenth warning. For polymorphic warnings, this decrease was 116.57 fixations, or $25.05 \%$ lower. This difference between static and polymorphic warnings was significant $\left(t_{(1816)}=2.295, p<.01, d=0.10\right)$. Second, for fixations on the warning text, static warnings decreased 104.87 fixations on average from the first warning to the tenth warning. For polymorphic warnings, this difference was 75.79 fixations, or $27.72 \%$ lower. The difference between static and polymorphic warnings was significant $\left(t_{(1813)}=\right.$ $3.44, p<.001, d=0.15)$. These findings corroborated the results of our hypothesis testing, which are summarized in Table 4.

\section{Table 4}


Summary of hypothesis testing.

\begin{tabular}{lc} 
Hypothesis & Supported? \\
\hline H1: Warning gaze duration will decrease over successive viewings per subject. & Yes \\
H2: Warning gaze duration will decrease more rapidly when viewing static warnings as compared & Yes \\
to polymorphic warnings. & Yes \\
H3: Warning text gaze duration will decrease over successive viewings per subject. & Yes \\
H4: Warning text gaze duration will decrease less rapidly when viewing polymorphic warnings as & \\
compared to static warnings. &
\end{tabular}

\section{Discussion}

This research makes several contributions, which we articulate in Table 5.

Table 5

Research contributions.

\begin{tabular}{|c|c|c|}
\hline Element of Research & Type & Contributions \\
\hline $\begin{array}{l}\text { Eye movement-based } \\
\text { memory (EMM) effect }\end{array}$ & $\begin{array}{l}\text { Theoretical, } \\
\text { methodological }\end{array}$ & $\begin{array}{l}\text { Presented the EMM effect as an explanation for how } \\
\text { habituation influences perceptions of security warnings. } \\
\text { Illustrated how the EMM effect may be used to } \\
\text { objectively measure habituation. }\end{array}$ \\
\hline Polymorphic warnings & Artifactual & $\begin{array}{l}\text { Designed a UI-design artifact, a polymorphic warning, } \\
\text { comprising nine graphical variations derived from the } \\
\text { warning science literature. }\end{array}$ \\
\hline $\begin{array}{l}\text { Polymorphic vs. static } \\
\text { warnings }\end{array}$ & Empirical, artifactual & $\begin{array}{l}\text { Demonstrated that polymorphic warnings are more } \\
\text { resistant to habituation than conventional warnings. }\end{array}$ \\
\hline $\begin{array}{l}\text { NeuroIS applied to } \\
\text { information security }\end{array}$ & $\begin{array}{l}\text { Theoretical, } \\
\text { methodological }\end{array}$ & $\begin{array}{l}\text { Highlighted the potential for applying NeuroIS theories } \\
\text { and methods to the domain of information security. }\end{array}$ \\
\hline
\end{tabular}

First, we contribute to the research by providing a direct measure of habituation to security warnings using the EMM effect. This neurophysiological measure allowed us to objectively measure

5 how habituation develops in response to repeated exposures to security messages. Our results show that attention to warnings drops precipitously after only the first exposure, with further decreases following subsequent exposures. Interestingly, we did not find a "floor" for the decrease in attention after ten exposures, suggesting that attention could have dropped even lower in our experiment if further repetitions had been conducted.

In addition, the EMM effect also provides insight into how habituation affects perceptions of warnings over time. The EMM literature explains that with repeated viewings, people unconsciously inspect a stimulus less than they do the first time it is viewed, instead relying on their memory of the image [72]. Our results confirm this finding of the neurobiological literature by showing that 
participants' visual sampling of warnings becomes substantially lower with successive viewings. This finding has serious implications for security warnings, which typically do not change their appearance except for textual variations in the message. Our results suggest that, similar to familiar wallpaper, repeated security warnings may be "seen" but no longer truly perceived.

Second, to address the problem of habituation, we developed a UI-design artifact - a polymorphic warning - which is a contribution in its own right [17]. Guided by the warning science literature, we identified nine graphical variations that research indicates should enhance attention to warnings. Our polymorphic warning design is practical in that it can be easily and generically applied to a wide range of security warnings. Whereas the frequent absence of the IT artifact in information systems research has been criticized [73, 74], our UI-design artifact is central to the contribution of this work.

Third, we demonstrated in our experiment that our polymorphic warning design is more resistant to habituation than conventional warnings, whose appearance do not change. Our analysis results showed that participants habituated to the polymorphic warnings at a significantly slower rate 15 compared to static warnings. Similarly, participants habituated less to the textual elements of the warnings over time compared to those of the static warnings, indicating a robust effect. Previous research on polymorphic warnings [10] did not examine their effects on habituation (either directly or indirectly), but instead examined risk-taking behavior. Therefore, it was previously unknown whether polymorphic warnings could reduce habituation. We addressed this research gap by establishing that polymorphic warnings reduce the occurrence of habituation significantly below that of conventional static warnings.

Fourth, our results answer the call of Crossler et al. [12] and Luo and Zhdanov [13] to apply NeuroIS methods to the domain of behavioral information security. Because many cognitive processes are automatic or unconscious, self-reported measures may not be effective in fully capturing these processes [75]. This paper shows that habituation to visual stimuli is one such 
process and demonstrates that habituation is obligatory and rapid in its onset, with a steep decrease in attention after only a few exposures. By applying eye tracking, we were able to fully capture this phenomenon. Given these results, it is likely that NeuroIS can be profitably applied to other security behaviors such as those related to fear appeals; password creation and management; and security 5 education, training, and awareness (SETA) programs, to name a few.

\subsection{Implications for Theory and Practice}

Although security researchers often consider users to be careless and inattentive [76], our results show that at least part of this behavior is obligatory and unconscious as a natural consequence of how the brain works. Our findings thus add to the chorus that users are not the enemy [77]—at least not consciously. These results also illustrate that users, in addition to having to defend against malicious actors and software, must cope with their own biology. Future research should investigate other obligatory, unconscious, or automatic behaviors that undermine the information security of individuals and organizations. NeuroIS methods are ideal for examining such behaviors because they can be used to detect phenomena beneath the cognition of the user [35].

Our findings have important implications for practice in the development of interventions to reduce habituation to security warnings. Rather than relying alone on interventions such as SETA programs, which encourage greater attention and vigilance $[78,79]$, our results suggest that an effective complementary technique is to develop UI-design artifacts that reduce habituation in the brain, such as the polymorphic warning developed in this study. In contrast to training that can

20 require hours or days, our polymorphic warning artifact requires no user training and elicits positive effects in milliseconds. In providing this benefit, our polymorphic warning design is unobtrusive and imposes no additional cost to the user. In contrast, other techniques for curbing habituation, such as imposing a time delay on security warnings before they can be dismissed $[9,10]$, impose a cost on 
the user that can be considerable over time and when aggregated over a large workforce or population [76].

Our polymorphic warning artifact is simple and cost-effective to implement in virtually any kind of system. With minimal additional graphical design and programming necessary to create a few

5 variations, polymorphic warnings can significantly decrease habituation to warnings. Because security warnings are relatively infrequent, the novelty effect of a few polymorphic variations may be sustained for a long period.

\subsection{Limitations}

This research is subject to several limitations. First, our experimental task required participants to view 200 images within approximately a 20-minute window. Because security warnings are relatively infrequent [80], our results may differ from habituation in actual practice. However, we chose this experimental design because it induced rapid and severe habituation, providing a more stringent test of the effectiveness of our polymorphic warning design. This is consistent with past investigations of habituation to software notifications and warnings [9, 30, 81]. Further, in a separate

15 study [82], we found that users do in fact habituate to a reasonable amount of warnings in a realistic context. Specifically, we designed a laboratory experiment in which participants were required to install and evaluate 20 Google Chrome web browser extensions in a 45-minute period on their own laptops. Because a permission warning is displayed whenever a Chrome extension is installed, participants also received 20 permission warnings. In this way, they were naturally habituated while

20 completing the task. We found that the pattern of habituation in this more realistic context was consistent with the overall pattern of habituation presented in this study. We therefore conclude that our results are still valid despite the artificiality introduced in our experimental design.

Second, and related to the previous point, our study used student participants. The object of laboratory experimentation is to maximize precision and control, not external validity [83, 84]. 
Accordingly, our sample was selected to ensure homogeneity among participants, reducing noise and thereby providing the strictest tests of the hypotheses [85]. Young people spend a comparatively large amount of time online [86] and frequently experience security threats; therefore, they represent a valid sample to study habituation of security warnings.

Third, although our study shows strong evidence that polymorphic warning design reduces habituation, we did not measure behavior. Attention to warnings will not always lead to good warning behavior, but it is a prerequisite. For this reason, our experiment focused on how to sustain attention to a warning — not on users' subsequent security behavior. Users may not care about a warning they attend to, but the opposite is worse: that users would care about the warning, but fail to notice it. We leave this question of how polymorphic warnings affect behavior in other contexts to future researchers.

Similarly, while eye-tracking tools allow us to measure habituation via fixations, using another neurophysiological tool such as EEG could help us understand the cognitive correlates of this habituation. Future research could use multiple methods, perhaps simultaneously, to capture

15 biological and cognitive responses to habituation. Finally, we used a cross-sectional design, and therefore we do not know how users habituate to security warnings that are repeated over extended periods of time. It is possible that over the course of weeks or months, the extent of habituation to security warnings may be severe. Future research is needed with longitudinal designs to examine the nature of habituation to warnings over time.

\section{Conclusion}

Although habituation to security warnings is understood to be a significant problem, previous studies have only indirectly examined this phenomenon. We contribute by using eye tracking to directly measure how habituation to security warnings occurs over time. Relying on the dual-process theory of habituation of neurobiology and the EMM effect, we explain that people unconsciously pay 
less attention to warnings previously seen as a function of memory. Using linear mixed effects

modeling on over 20,000 observations, we demonstrate that this effect of habituation sets in rapidly and progresses with successive exposures to warnings. We also show that our polymorphic warning design significantly reduces the rate of habituation, offering a substantial improvement over the status quo. Beyond the problem of habituation, our findings demonstrate the value of applying

NeuroIS methods to the domain of behavioral information security, and point to a number of avenues for future research.

\section{Acknowledgements}

This work was supported by the National Science Foundation under Grants CNS-XXXXXXX and DGE-XXXXXXX.

\section{References}

[1] C. Drew, Stolen data is tracked to hacking at lockheed, The New York Times, 2011.

[2] K. Poulsen, Second defense contractor 1-3 'actively targeted' with rsa securid hacks, Wired, 2011.

[3] M. Hyppönen, How we found the file that was used to hack rsa, 2011. http://www.f(4).com/weblog/archives/00002226.html. Accessed 8/20/14.

[4] C. Bravo-Lillo, L.F. Cranor, J. Downs, S. Komanduri, M. Sleeper, Improving computer security dialogs, in: P. Campos, N. Graham, J. Jorge, N. Nunes, P. Palanque, M. Winckler (Eds.), Proceedings of the 13th IFIP TC 13 International Conference on Human-Computer Interaction - Volume 6949 Part IV, SpringerVerlag, Lisbon, Portugal, 2011, pp. 18-35.

20 [5] J. Sunshine, S. Egelman, H. Almuhimedi, N. Atri, L.F. Cranor, Crying wolf: An empirical study of ssl warning effectiveness, SSYM'09 Proceedings of the 18th conference on USENIX security symposium, Montreal, Canada, 2009, pp. 399-416.

[6] A. Sotirakopoulos, K. Hawkey, K. Beznosov, On the challenges in usable security lab studies: Lessons learned from replicating a study on ssl warnings, Proceedings of the Seventh Symposium on Usable Privacy and Security (SOUPS), ACM, Menlo Park, CA, 2011, pp. 3:1-3:18.

[7] D. Akhawe, A.P. Felt, Alice in warningland: A large-scale field study of browser security warning effectiveness, Proceedings of the 22nd USENIX Conference on Security, USENIX Association, Washington, D.C., 2013, pp. 257-272.

[8] P.M. Groves, R.F. Thompson, Habituation: A dual-process theory, Psychological Review, 77 (1970) 419-450.

[9] C. Bravo-Lillo, S. Komanduri, L.F. Cranor, R.W. Reeder, M. Sleeper, J. Downs, S. Schechter, Your attention please: Designing security-decision uis to make genuine risks harder to ignore, Proceedings of the Ninth Symposium on Usable Privacy and Security ACM, Newcastle, United Kingdom, 2013, pp. 1-12. [10] J.C. Brustoloni, R. Villamarín-Salomón, Improving security decisions with polymorphic and 35 audited dialogs, Proceedings of the Third symposium on Usable Privacy and Security (SOUPS 2007), ACM, New York, NY, USA, 2007, pp. 76-85. 
[11] A.P. Felt, E. Ha, S. Egelman, A. Haney, E. Chin, D. Wagner, Android permissions: User attention, comprehension, and behavior, Proceedings of the Eighth Symposium on Usable Privacy and Security, ACM, 2012, pp. 3:1-3:14.

[12] R.E. Crossler, A.C. Johnston, P.B. Lowry, Q. Hu, M. Warkentin, R. Baskerville, Future directions for behavioral information security research, Computers \& Security, 32 (1) (2013) 90-101.

[13] X. Luo, D. Zhdanov, Call for papers: A comprehensive perspective on information systems security: Technical advances and behavioral issues, Decision Support Systems, 2015.

http://www.journals.elsevier.com/decision-support-systems/call-for-papers/comprehensive-perspective-oninformation-systems-security. Accessed 8/20/15.

[14] J.D. Ryan, R.R. Althoff, S. Whitlow, N.J. Cohen, Amnesia is a deficit in relational memory, Psychological Science, 11 (6) (2000) 454-461.

[15] D.E. Hannula, R.R. Althoff, D.E. Warren, L. Riggs, N.J. Cohen, J.D. Ryan, Worth a glance: Using eye movements to investigate the cognitive neuroscience of memory, Frontiers in Human Neuroscience, 4 (2010) 166.

[17] A. Vance, P.B. Lowry, D. Eggett, Increasing accountability through user-interface design artifacts: A new approach to address the problem of access-policy violations, MIS Quarterly, 39 (2) (2015) 345-366.

[18] R.F. Thompson, W.A. Spencer, Habituation: A model phenomenon for the study of neuronal substrates of behavior, Psychological Review, 73 (1) (1966) 16-43.

[19] C.H. Rankin, Introduction to special issue of neurobiology of learning and memory on habituation, Neurobiology of Learning and Memory, 92 (2) (2009) 125-126.

[20] M.Ö. Çevik, Habituation, sensitization, and pavlovian conditioning, Frontiers in Integrative Neuroscience, 8 (2014) 13.

[21] S. Schmid, D.A. Wilson, C.H. Rankin, Habituation mechanisms and their importance for cognitive function, Frontiers in Integrative Neuroscience, 8 (2014) 97.

[22] J. Colombo, D.W. Mitchell, Infant visual habituation, Neurobiology of Learning and Memory, 92 (2) (2009) 225-234.

[23] B. Verplanken, H. Aarts, Habit, attitude, and planned behaviour: Is habit an empty construct or an interesting case of goal-directed automaticity?, European Review of Social Psychology, 10 (1) (1999) 101 134.

[24] B. Verplanken, S. Orbell, Reflections on past behavior: A self-report index of habit strength, Journal of Applied Social Psychology, 33 (6) (2003) 1313-1330.

[25] M. Ramaswami, Network plasticity in adaptive filtering and behavioral habituation, Neuron, 82 (6) (2014) 1216-1229.

[26] C.H. Rankin, T. Abrams, R.J. Barry, S. Bhatnagar, D.F. Clayton, J. Colombo, G. Coppola, M.A. Geyer, D.L. Glanzman, S. Marsland, F.K. McSweeney, D.A. Wilson, C.-F. Wu, R.F. Thompson, Habituation revisited: An updated and revised description of the behavioral characteristics of habituation, Neurobiology of Learning and Memory, 92 (2) (2009) 135-138.

[27] S.E. Schechter, R. Dhamija, A. Ozment, I. Fischer, The emperor's new security indicators, Security and Privacy, 2007. SP'07. IEEE Symposium on, IEEE, Berkeley, CA, 2007, pp. 51-65.

[28] D. Sharek, C. Swofford, M. Wogalter, Failure to recognize fake internet popup warning messages, Proceedings of the Human Factors and Ergonomics Society Annual Meeting, Sage Publications, New York, New York, 2008, pp. 557-560.

[29] M.S. Wogalter, Purposes and scope of warnings, in: M.S. Wogalter (Ed.) Handbook of warnings, Lawrence Erlbaum Associates, 2006, pp. 3-9.

[30] C. Bravo-Lillo, L. Cranor, S. Komanduri, S. Schechter, M. Sleeper, Harder to ignore? Revisiting pop-up fatigue and approaches to prevent it, USENIX Association, 2014, pp. 105-111.

[31] S. Shimojo, C. Simion, E. Shimojo, C. Scheier, Gaze bias both reflects and influences preference, Nature Neuroscience, 6 (12) (2003) 1317-1322.

50 [32] K. Rayner, Eye movements in reading and information processing: 20 years of research, Psychological Bulletin, 124 (3) (1998).

[33] I. Benbasat, A. Dimoka, P.A. Pavlou, L. Qiu, Incorporating social presence in the design of the anthropomorphic interface of recommendation agents: Insights from an fmri study, ICIS 2010 Proceedings, AIS, St. Louis, 2010. 
[35] A. Dimoka, R.D. Banker, I. Benbasat, F.D. Davis, A.R. Dennis, D. Gefen, A. Gupta, A. Lschebeck, P.H. Kenning, P.A. Pavlou, G. Muller-Putz, R. Riedl, J. vom Brocke, B. Weber, On the use of neurophysiological tools in is research: Developing a research agenda for neurois, MIS Quarterly, 36 (3) (2012) 679-702.

[36] C.N. Smith, L.R. Squire, Experience-dependent eye movements reflect hippocampus-dependent (aware) memory, The Journal of Neuroscience, 28 (48) (2008) 12825-12833.

[37] J.H. Goldberg, M.J. Stimson, M. Lewenstein, N. Scott, A.M. Wichansky, Eye tracking in web search tasks: Design implications, Proceedings of the 2002 symposium on Eye tracking research \& applications, ACM, New Orleans, Louisiana, 2002, pp. 51-58.

[38] D. Cyr, M. Head, H. Larios, B. Pan, Exploring human images in website design: A multi-method approach, MIS Quarterly, 33 (3) (2009).

[39] T.O. Meservy, M.L. Jensen, K.J. Fadel, Evaluation of competing candidate solutions in electronic networks of practice, Information Systems Research, 25 (1) (2014) 15-34.

[40] P.-M. Léger, S. Sénecal, F. Courtemanche, A. Ortiz de Guinea, R. Titah, M. Fredette, È. LabonteLemoine, Precision is in the eye of the beholder: Application of eye fixation-related potentials to information systems research, Journal of the Association for Information Systems, 15 (10) (2014) 651-678.

[41] S. Djamasbi, T. Tullis, M. Siegel, D. Capozzo, R. Groezinger, F. Ng, Generation y \& web design: Usability through eye tracking, Proceedings of America's Conference on Information Systems, Toronto, Canada, 2008, p. 77.

20 [42] R.J.K. Jacob, K.S. Karn, Eye tracking in human-computer interaction and usability research: Ready to deliver the promises, in: J. Hyona, R. Radach, H. Deubel (Eds.) The mind's eye: Cognitive and applied aspects of eye movement research, Elsevier, 2003, pp. 573-605.

[43] S.P. Liversedge, J.M. Findlay, Saccadic eye movements and cognition, Trends in cognitive sciences, 4 (1) (2000) 6-14.

[44] M. Hayhoe, D. Ballard, Eye movements in natural behavior, Trends in cognitive sciences, 9 (4) (2005) 188-194.

[45] J.J. Heisz, D.I. Shore, More efficient scanning for familiar faces, Journal of Vision, 8 (1) (2008).

[46] J.J. Heisz, J.D. Ryan, The effects of prior exposure on face processing in younger and older adults, Frontiers in Aging Neuroscience, 3 (2011) 15.

30 [47] E. Sokolov, Higher nervous functions: The orienting reflux, Annual Review of Physiology, 25 (1963) 545-580.

[48] L.R. Squire, C.E. Stark, R.E. Clark, The medial temporal lobe, Annual Review of Neuroscience, 27 (2004) 279-306.

[49] M.G. Packard, B.J. Knowlton, Learning and memory functions of the basal ganglia, Annual Review of Neuroscience, 25 (1) (2002) 563-593.

[50] K. Grill-Spector, R. Henson, A. Martin, Repetition and the brain: Neural models of stimulusspecific effects, Trends in cognitive sciences, 10 (1) (2006) 14-23.

[51] K.A. Norman, R.C. O'Reilly, Modeling hippocampal and neocortical contributions to recognition memory: A complementary-learning-systems approach, Psychological Review, 110 (4) (2003) 611-646. [52] M.A. Yassa, S.M. Stark, A. Bakker, M.S. Albert, M. Gallagher, C.E. Stark, High-resolution structural and functional mri of hippocampal ca3 and dentate gyrus in patients with amnestic mild cognitive impairment, NeuroImage, 51 (3) (2010) 1242-1252.

[53] C.B. Kirwan, M.I. Nash, Resolving interference: The role of the human hippocampus in pattern separation, in: P.A. Jackson, A.A. Chiba, R.F. Berman, M.E. Ragozzino (Eds.) The neurobiological basis of memory, Springer, 2016, pp. 151-173.

[54] A. Dimoka, P.A. Pavlou, F.D. Davis, Research commentary-neurois: The potential of cognitive neuroscience for information systems research, Information Systems Research, 22 (4) (2011) 687-702.

[55] C.C. Braun, B. Greeno, N.C. Silver, Differences in behavioral compliance as a function of warning color, Proceedings of the Human Factors and Ergonomics Society Annual Meeting, Sage

$50 \quad$ Publications, 1994, pp. 379-383.

[56] K.R. Laughery, S.L. Young, K.P. Vaubel, J.W. Brelsford Jr, The noticeability of warnings on alcoholic beverage containers, Journal of Public Policy \& Marketing, 12 (1) (1993) 38-56.

[57] J.A. Strawbridge, The influence of position, highlighting, and imbedding on warning effectiveness, Proceedings of the Human Factors and Ergonomics Society Annual Meeting, Sage, Dayton, OH, 1986, pp. 716-720. 
[58] S.L. Young, M. Wogalter, Effects of conspicuous print and pictorial icons on comprehension and memory of instruction manual warnings, Human Factors, 32 (1990) 637-649.

[59] M.J. Kalsher, M.S. Wogalter, B.M. Racicot, Pharmaceutical container labels: Enhancing preference perceptions with alternative designs and pictorials, International Journal of Industrial Ergonomics, 18 (1) (1996) 83-90.

[60] R.J. Sojourner, M.S. Wogalter, The influence of pictorials on evaluations of prescription medication instructions, Drug Information Journal, 31 (3) (1997) 963-972.

[61] M.J. Kalsher, B.M. Brewster, M.S. Wogalter, M.E. Spunar, Hazard level perceptions of current and proposed warning sign and label panels, Proceedings of the Human Factors and Ergonomics Society Annual Meeting, 39 (5) (1995) 351-355.

[62] N.C. Silver, M.S. Wogalter, Broadening the range of signal words, Proceedings of the Human Factors and Ergonomics Society Annual Meeting, Sage, Denver, Colorado, 1989, pp. 555-559.

[63] C.C. Braun, N.C. Silver, Interaction of signal word and colour on warning labels: Differences in perceived hazard and behavioural compliance, Ergonomics, 38 (11) (1995) 2207-2220.

[64] C.M. Rudin-Brown, M.P. Greenley, A. Barone, J. Armstrong, A.F. Salway, B.J. Norris, The design of child restraint system (crs) labels and warnings affects overall crs usability, Traffic Injury Prevention, 5 (1) (2004) 8-17.

[65] M.S. Sanders, E.J. McCormick, Human factors in engineering and design, 7th ed., McGraw-Hill, 1987.

[66] S.L. Young, Increasing the noticeability of warnings: Effects of pictorial, color, signal icon and border, Proceedings of the Human Factors and Ergonomics Society Annual Meeting, Sage, San Francisco, VA, 1991, pp. 580-584.

[67] W.J. Vigilante, M. Wogalter, Effects of label format on knowledge acquisition and perceived readability by younger and older adults, Ergonomics, 46 (4) (2003) 327-344.

[68] M. Wogalter, W.J. Vigilante Jr, Attention switch and maintenance, in: M.S. Wogalter (Ed.) Handbook of warnings, Lawrence Erlbaum Associates, 2006, pp. 245-266.

[69] R. Riedl, P.-M. Léger, Fundamentals of neurois: Information systems and the brain, Springer,

2016.

[70] D. Straub, M.-C. Boudreau, D. Gefen, Validation guidelines for is positivist research, Communications of the Association for Information Systems, 13 (24) (2004) 380-427.

[71] D. Bates, M. Maechler, B. Bolker, S. Walker, Fitting linear mixed-effects models using lme4, Journal of Statistical Software, 67 (1) (2015) 1-48.

[72] E.F. Chua, D.E. Hannula, C. Ranganath, Distinguishing highly confident accurate and inaccurate memory: Insights about relevant and irrelevant influences on memory confidence, Memory, 20 (1) (2011) 48-

[73] S. Akhlaghpour, J. Wu, L. Lapointe, A. Pinsonneault, The ongoing quest for the it artifact: Looking back, moving forward, Journal of Information Technology, 28 (2) (2013) 150-166.

[74] W.J. Orlikowski, C.S. Iacono, Research commentary: Desperately seeking the "it" in it researcha call to theorizing the it artifact, Information Systems Research, 12 (2) (2001) 121-134.

[75] A. Dimoka, I. Benbasat, K. Lim, D. Straub, E. Walden, Neurois: Challenges and solutions, Proceedings of the 2010 International Conference on Information Systems, St. Louis, Missouri, 2010.

[76] C. Herley, So long, and no thanks for the externalities: The rational rejection of security advice by users, Proceedings of the 2009 Workshop on New Security Paradigms, ACM, 2009, pp. 133-144.

[77] A. Adams, M.A. Sasse, Users are not the enemy, Communications of the ACM, 42 (12) (1999) $40-46$.

[78] M. Karjalainen, M. Siponen, Toward a new meta-theory for designing information systems (is) security training approaches, Journal of the Association for Information Systems, 12 (8) (2011) 518-555.

[79] J. D'Arcy, A. Hovav, D. Galletta, User awareness of security countermeasures and its impact on information systems misuse: A deterrence approach, Information Systems Research, 20 (1) (2009) 79-98.

[80] A.P. Felt, A. Ainslie, R.W. Reeder, S. Consolvo, S. Thyagaraja, A. Bettes, H. Harris, J. Grimes, Improving ssl warnings: Comprehension and adherence, Proceedings of the Conference on Human Factors in Computing Systems, ACM, Seoul, South Korea, 2015.

[81] T.S. Amer, J.-M.B. Maris, Signal words and signal icons in application control and information technology exception messages - hazard matching and habituation effects, Journal of Information Systems, 21 (2) (2007) 1-25. 
[82] Authors, Title and publication outlet withheld to preserve the anonymity of the review process. (2015).

[83] A.R. Dennis, J.S. Valacich, Conducting experimental research in information systems, Communications of the Association for Information Systems, 7 (5) (2001).

[84] J.E. McGrath, Dilemmatics: The study of research choices and dilemmas, American Behavioral Scientist, 25 (2) (1981) 179-210.

[85] B.J. Calder, L.W. Phillips, A.M. Tybout, The concept of external validity, Journal of Consumer Research, 9 (3) (1982) 240-244.

[86] Pew Research Center, Internet use and home broadband connections, Pew Internet \& American Life Project, 2012. 